\title{
Small impacts on the giant planet Jupiter
}

\author{
R. Hueso ${ }^{1}$, M. Delcroix ${ }^{2}$, A. Sánchez-Lavega ${ }^{1}$, S. Pedranghelu ${ }^{3}$, G. Kernbauer ${ }^{4}$, J. McKeon ${ }^{5}$, A. Fleckstein ${ }^{6}$, \\ A. Wesley ${ }^{7}$, J. M. Gómez-Forrellad ${ }^{8}$, J. F. Rojas ${ }^{1}$, and J. Juaristi ${ }^{1}$ \\ ${ }^{1}$ Física Aplicada I, Escuela de Ingeniería de Bilbao, UPV/EHU, Alameda Urquijo s/n, 48013 Bilbao, Spain \\ e-mail: ricardo.hueso@ehu.es \\ ${ }^{2}$ Societé Astronomique de France, Paris, France \\ 3 Amateur Astronomer, Lycée Jules Antonini, Ajaccio; Chemin des vignes, 20167 Afa, France \\ ${ }^{4}$ Amateur Astronomer, Salurnergasse 9/6, 2340 Mödling, Austria \\ ${ }^{5}$ Meath Astronomy Group, Dublin, Ireland \\ ${ }^{6}$ Amateur Astronomer, Gablonzerstraße 33, 38259 Salzgitter, Germany \\ 7 Astronomical Society of Australia, 1502 Rubyvale Rd, Rubyvale, Queensland 4702, Australia \\ ${ }^{8}$ Fundacio Observatori Esteve Duran, Seva, Spain
}

Received 23 January 2018 / Accepted 3 April 2018

\begin{abstract}
Context. Video observations of Jupiter obtained by amateur astronomers over the past 8 years have shown five flashes of light with durations of $1-2 \mathrm{~s}$, each observed by at least two observers that were geographically separated. The first three of these events occurred on 3 June 2010, 20 August 2010, and 10 September 2012. Previous analyses of their light curves showed that they were caused by the impact of objects of 5-20 m in diameter, depending on their density, with a released energy comparable to superbolides on Earth of the class of the Chelyabinsk airburst. The most recent two flashes on Jupiter were detected on 17 March 2016 and 26 May 2017 and are analyzed here.

Aims. We characterize the energy involved together with the masses and sizes of the objects that produced these flashes. The rate of similar impacts on Jupiter provides improved constraints on the total flux of impacts on the planet, which can be compared to the amount of exogenic species detected in the upper atmosphere of Jupiter.

Methods. We extracted light curves of the flashes and calculated the masses and sizes of the impacting objects after calibrating each video observation. We also present results from a systematic search of impacts on $>72000$ video amateur observations with a customized software that is based on differential photometry of the images. An examination of the number of amateur observations of Jupiter as a function of time over the past years allows us to interpret the statistics of these impact detections.

Results. The cumulative flux of small objects (5-20 m or larger) that impact Jupiter is predicted to be low (10-65 impacts per year), and only a fraction of them are potentially observable from Earth (4-25 observable impacts per year in a perfect survey). These numbers imply that many observers are required to efficiently discover Jupiter impacts.

Conclusions. We predict that more impacts will be found in the next years, with Jupiter opposition displaced toward summer in the northern hemisphere where most amateur astronomers observe. Objects of this size contribute negligibly to the abundance of exogenous species and dust in the stratosphere of Jupiter when compared with the continuous flux from interplanetary dust particles punctuated by giant impacts. Flashes of a high enough brightness (comparable at their peak to a +3.3 magnitude star) could produce an observable debris field on the planet. We estimate that a continuous search for these impacts might find these events once every $0.4-2.6 \mathrm{yr}$.
\end{abstract}

Key words. planets and satellites: atmospheres - planets and satellites: individual: Jupiter - meteorites, meteors, meteoroids

\section{Introduction}

Because of its large gravitational attraction and effective cross section, the giant planet Jupiter is the most likely place to receive impacts in the solar system. Direct and dramatic evidence of impacts on Jupiter was acquired with the observations of the series of impacts from the comet Shoemaker-Levy 9 (SL9) in July 1994 (Harrington et al. 2004). On 19 July 2009, an unknown body collided with Jupiter on its night side (Sánchez-Lavega et al. 2010). In both cases, the impacts produced large spots of material that were dark in the visible wavelength range and bright in methane absorption bands because of the high altitude of the debris fields. These spots remained visible for weeks to years (Hammel et al. 1995, 2010; Sánchez-Lavega et al. 1998, 2011).

Impacts supply disequilibrium species to the upper atmosphere of Jupiter, which in the case of SL9 are still unambiguously observable today because of the higher concentration of water, $\mathrm{CO}$, and other chemical species in the southern hemisphere of Jupiter (Lellouch et al. 2002; Cavalié et al. 2013). Spectroscopic observations of the planet allow inferring the amount of exogenic molecules in the upper atmosphere of Jupiter. However, the relative contribution to the abundance of exogenic water and carbon dioxide from impacts of very different size range from a continuous supply of interplanetary dust to rare impacts of large objects are not yet well characterized (Lellouch et al. 2002; Bézard et al. 2002; Poppe 2016; Moses \& Poppe 2017).

Impacts from objects of about $10 \mathrm{~m}$ in diameter have been detected in telescopic observations of Jupiter from the sudden release of luminous energy when the impacting objects enter the atmosphere of Jupiter and explode as atmospheric bolides (Hueso et al. 2010b, 2013). Five impacts have been detected in this way since 2010. Three of them have been examined 
previously in the literature (Hueso et al. 2010b, 2013), and two more have occurred since then. Each of these impacts has been detected simultaneously by more than one observer (12 observers recorded 11 video acquisitions for a total of five impact bolides).

Hueso et al. (2010b) presented the analysis of the first bolide impact on Jupiter and the general method for calibrating light curves from amateur observations of Jovian flashes. They also converted them into energies, masses, and sizes of the impacting object. The first Jovian bolide was caused by an object with a diameter in the range of $10 \mathrm{~m}$, releasing an energy comparable to an object of about $30 \mathrm{~m}$ impacting Earth's atmosphere. Followup observations with telescopes such as the Very Large Telescope (VLT) or the Hubble Space Telescope (HST) showed no evidence of atmospheric debris left by the impact, confirming the small size of the object. Hueso et al. (2013) extended this study to a characterization of the three known bolides in 2013. The combined analysis of these three impacts allowed a first quantification of the flux of similar impacts on Jupiter. The expected number was 12-60 impacts per year for objects larger than 5$20 \mathrm{~m}$ in diameter. This impact rate is close to expectations based on an extrapolation of dynamical models of comets and asteroids in orbits prone to Jupiter encounters (Levison et al. 2000; 30-100 collisions per year for objects with diameters larger than 5-20 m). Hueso et al. (2013) also presented model simulations of airbursts caused by these small-sized objects following similar techniques to those used in simulations of larger impacts (Korycansky et al. 2006; Palotai et al. 2011; Pond et al. 2012).

We here update previous results presented in Hueso et al. (2010b, 2013) with the analysis of the latest two impacts detected in March 2016 and May 2017. We examine how the new observations constrain the flux of impacts on Jupiter similarly to the estimate in Hueso et al. (2013). We also discuss the implications of the predicted flux of impacts on the amount of exogenic species (water and carbon monoxide) and dust in the upper atmosphere of Jupiter, and we discuss the probability of finding more intense flashes from larger objects that could leave observable traces in the atmosphere of Jupiter from follow-up observations.

The outline of this paper is the following: in Sect. 2 we summarize previous observations of the first three impact fireballs (Hueso et al. 2010b, 2013), and we give observational details of the latest two impacts found on Jupiter in March 17, 2016, and May 26, 2017. In Sect. 3 we present light curves of these two impacts and calibrate the images to obtain size and mass estimations of these objects. In Sect. 4 we examine the sizes and masses of impacts required to leave an observable debris field in the planet atmosphere. In Sect. 5 we discuss current efforts of detecting new observation flashes on Jupiter. In Sect. 6 we present an statistical analysis of the amateur observations to infer the statistical significance of these impact detections in a larger context. In Sect. 7 we present an updated estimate of the impact flux on Jupiter and discuss the implications for exogenous water and carbon monoxide on the upper atmosphere of Jupiter and the probability of finding observable debris fields in the atmosphere of Jupiter after more intense superbolides. We present our conclusions and a summary of our findings in Sect. 8.

\section{Observations}

\subsection{Summary of previous impacts}

On June 3, 2010, Anthony Wesley (Australia) and Christopher Go (Philippines) recorded a short flash while taking video observations of Jupiter. The flash lasted about two seconds and was observed by A. Wesley, who issued an e-email alert that was confirmed later by C. Go. A quick and large follow-up campaign was organized, and it obtained observations of the planet within a few days with telescopes such as the VLT and HST. None of these observations found any debris field in the region hit by the impact. A later analysis of the flash light curve resulted in the conclusion that it was caused by an object of $8-13 \mathrm{~m}$ in diameter impacting the atmosphere of Jupiter and producing a giant fireball (Hueso et al. 2010b).

Two months later, on August 20, 2010, another flash on Jupiter was detected by amateur astronomer Masayuki Tachikawa of Japan, and it was confirmed by Kazuo Aoki and Masayuki Ishimaru. This flash was found in videos of lower quality in the wake of published news in amateur astronomy journals about the first flash. Two years later, on September 10, 2012, a new fireball on Jupiter was discovered by a visual observer, who issued an alert in astronomical forums (Dan Petersen from Racine in Wisconsin). The flash was later confirmed by a video observation obtained by George Hall from Dallas, Texas. This flash was significantly brighter than the previous two impacts. Subsequent analysis of the video observation agreed remarkably well with the brightness estimate from Dan Petersen, who had observed the flash visually in the telescope eyepiece.

Figure 1 shows processed versions of the observations of the three flashes. In these three cases (as well as in the next two flashes discussed below), an observer raised the alert to the amateur community after observing the impact flash. Confirmations from other observers who had been taking data at the same time quickly followed, but we remark that most observers did not see the flash originally when they were at the telescope, or when they first analyzed their video observations with automatic stacking software tools. Amateur astronomers combine thousands of frames from a single video into a stacked image with high signal-to-noise ratio using automatic software tools (Mousis et al. 2014), where the light of any possible short flash dilutes within the rest of the frames, rendering it invisible in the final image. In all cases, the regions that were hit did not show any trace of the bolide material in later observations either by large telescopes such as in June 2010 and September 2012 or by fast amateur follow-ups in August 2010. Therefore, these events can only be discovered if it is spotted in the few seconds during which each impact produces a bright fireball.

\subsection{Impact on March 17, 2016}

A new impact was detected on March 17, 2016, by Gerrit Kernbauer (Austria). The impact was announced ten days after the observation because the relatively poor seeing of that night caused G.K. to delay an analysis of the video observations. The announcement was noted by John McKeon (Ireland), who had been observing the planet in the same night for $3.5 \mathrm{~h}$, building a time-lapse video of Jupiter and its moons. The second video confirmed the finding with better image quality. It is difficult to visually find a short flash of light of $2 \mathrm{~s}$ in a sequence of video observation that lasts several hours.

\subsection{Impact on May 26, 2017}

The most recent impact on Jupiter was found by Sauveur Pedranghelu from Corsica (France) on May 26, 2017. The impact was announced the next day and was quickly confirmed by two German observers, Thomas Riessler and André Fleckstein, both after reading news of the impact posted on German astronomical forums. The videos by S.P. and T.R. were of excellent quality, 

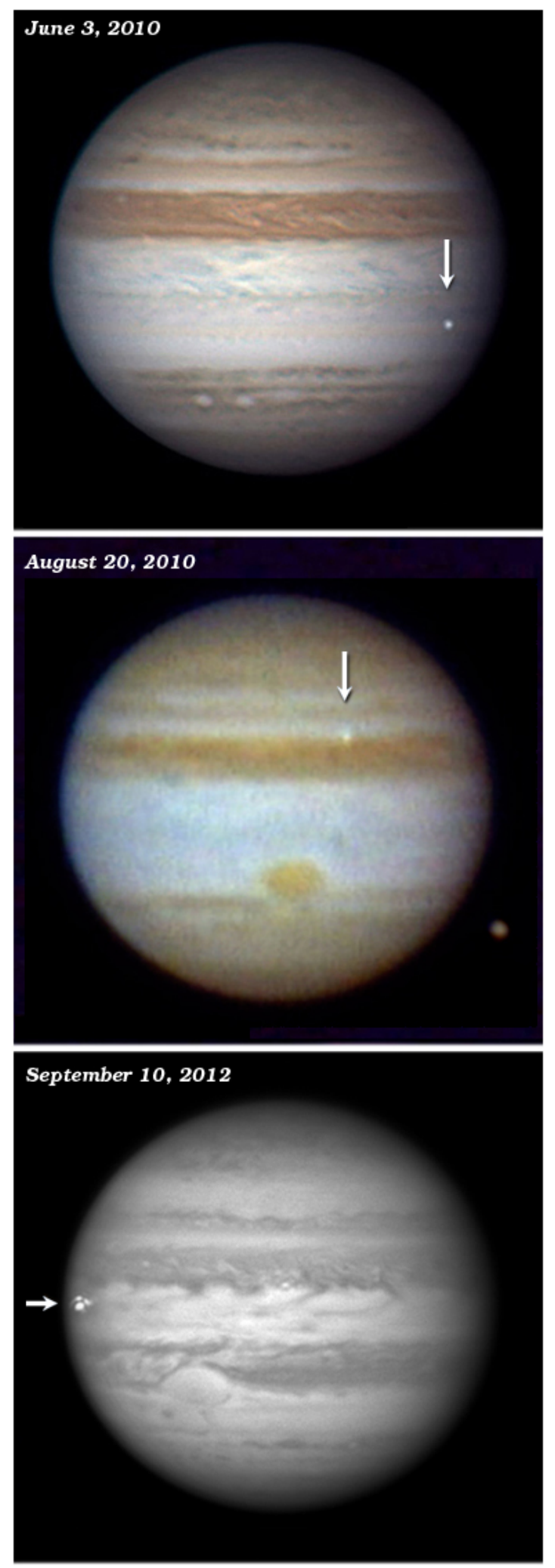

Fig. 1. First three fireballs found on Jupiter from observations by A. Wesley (top panel), M. Tachikawa (middle panel), and G. Hall (bottom panel). Arrows show the position of the impacts. Images have been processed by stacking the frames where the flash is visible and adding the result over a Jupiter image built from the stack of the full video of the planet at the time of the impact. The color in the first panel comes from acquisitions obtained with a filter wheel in the minutes before and after the flash. The color in the second panel comes from the detector, which makes use of a Bayer mask to produce color images. The diffraction-like ring patterns in the last panel show the brightest flash.
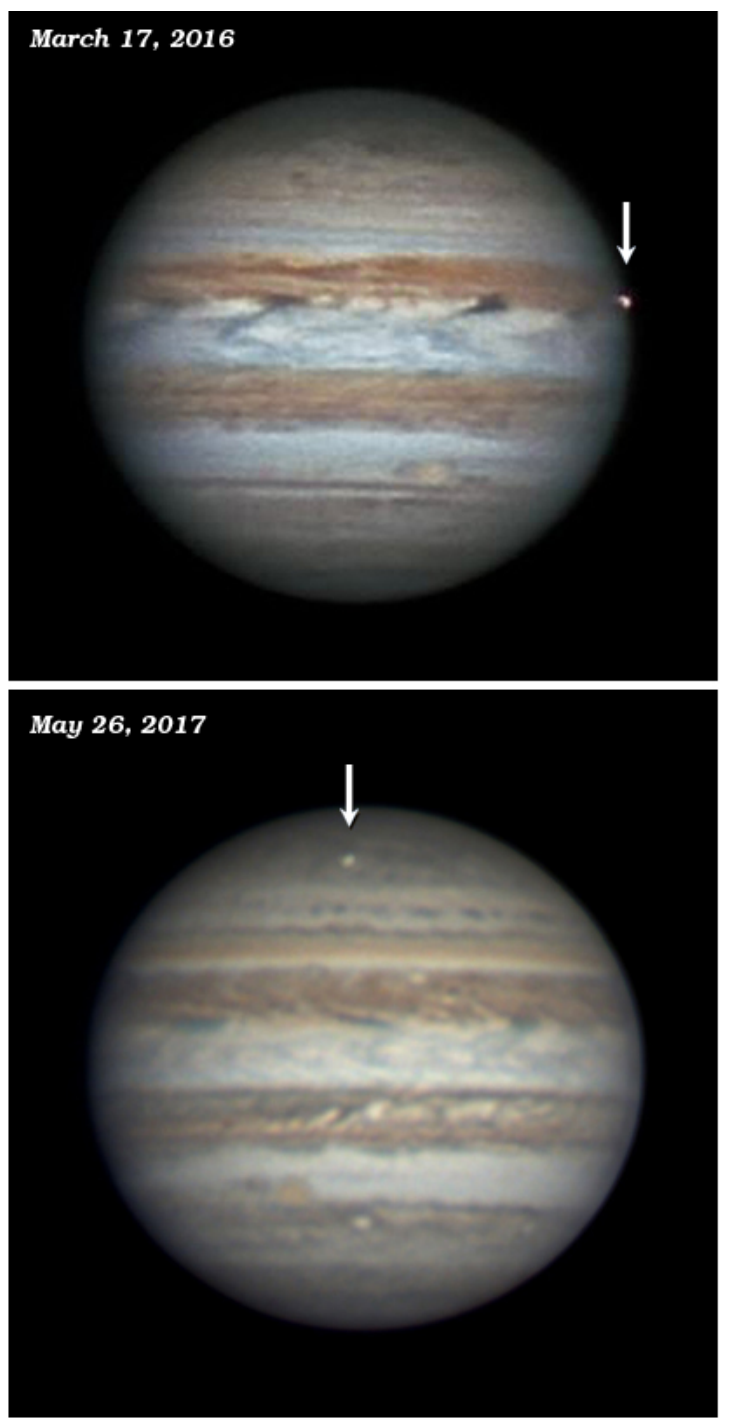

Fig. 2. Latest two fireballs found on Jupiter. Arrows show the position of the impacts. Upper panel: impact on March 17, 2016, observed by G. Kernbauer and J. McKeon. The image has been processed for aesthetics by S. Voltmer using data from the two video observations. The color comes from the G.K. video and the luminance from J. McKeon. Bottom panel: impact on May 26, 2017, observed by S. Pedranghelu, T. Riessler, and A. Fleckstein. The image is a combination of video observations in color from S.P. and T.R. All image combinations were done with stacking software and wavelet processing to increase the sharpness of atmospheric features, and the impact was added from a separate processing of the frames where the flash is visible.

and the video observation by A.F. was not as good because of the atmospheric seeing at his location. It was almost impossible to observe the flash in this video without previous knowledge of the moment where the impact had occurred, meaning that good atmospheric seeing is a critical factor in the discoveries of these events. Figure 2 shows these two impacts from a variety of video observations. Table 1 summarizes the dates of all the impacts, observers, and equipment.

\subsection{Follow-up observations}

For the 2016 and 2017 impacts, observations by a variety of amateur astronomers were obtained from $10 \mathrm{~min}$ to a few rotations after the impact. None of these observations showed any debris field on the surface of the planet at the locations of each 
Table 1. Jovian bolides detections.

\begin{tabular}{|c|c|c|c|c|c|c|}
\hline $\begin{array}{l}\text { Date (yyyy-mm-dd) } \\
\text { Time (hh-mm-ss) } \\
\text { (UT) }\end{array}$ & $\begin{array}{l}\text { Observers } \\
\text { (and locations) }\end{array}$ & $\begin{array}{l}\text { Telescope } \\
\text { diameter } \\
(\mathrm{cm})\end{array}$ & Detector & Filters & $\begin{array}{c}\text { Flash } \\
\text { duration } \\
(\mathrm{s})\end{array}$ & $\begin{array}{c}\text { Sampling } \\
\text { rate } \\
(\mathrm{fps})^{*} \\
\end{array}$ \\
\hline $2010-06-03$ & A.Wesley (Australia) & 37 & Point Grey Flea3 & $650 \mathrm{~nm}$ & 1.9 & 60 \\
\hline$(20: 31: 20)$ & C. Go (Phillipines) & 28 & Point Grey Flea3 & $435 \mathrm{~nm}$ & 0.95 & 55 \\
\hline $2010-08-20$ & M. Tachikawa (Japan) & 15 & Philips ToUCam II & Bayer RGB & 1.4 & 30 \\
\hline \multirow[t]{2}{*}{$(18: 21: 56)$} & K. Aoki (Japan) & 23.5 & Philips ToUCam II & Bayer RGB & 1.9 & 15 \\
\hline & M. Ishimaru (Japan) & 12.5 & Philips ToUCam II & Bayer RGB & 1.1 & 30 \\
\hline 2012-09-10 & D. Petersen (USA) & 30.5 & Visual observation & - & - & - \\
\hline$(11: 35: 30)$ & G. Hall (USA) & 30.5 & Point Grey Flea3 & $640 \mathrm{~nm}$ & 1.7 & 15 \\
\hline 2016-03-17 & G. Kernbauer (Austria) & 20 & QHY5LII & Bayer RGB & 1.15 & 47 \\
\hline$(00: 18: 39)$ & J. McKeon (Ireland) & 28 & ASI120MM & IR742 & 1.30 & 26 \\
\hline $2017-05-26$ & S. Pedranghelu (France) & 20.3 & ASI224MC & Bayer RGB & 1. 38 & 61.79 \\
\hline \multirow{2}{*}{$(19: 24: 50)$} & T. Riessler (Germany) & 20.3 & ASI120MC & Bayer RGB & 0.88 & 30.78 \\
\hline & A. Fleckstein (Germany) & 28 & ASI120MM & IR742 & 0.92 & 30 \\
\hline
\end{tabular}

Notes. ${ }^{(*)}$ fps stands for frames per second.

impact. We surveyed the Planetary Virtual Observatory and Laboratory (PVOL; Hueso et al. 2010a, 2018) and the Association of Lunar and Planetary Observers (ALPO) Japan databases of amateur observations as well as several amateur astronomical forums in search of images covering these areas. In the case of the March 2016 impact, the impact area was just disappearing behind the east limb, and the high-resolution observation closest in time was obtained by Randy Christensen (USA) one Jovian rotation later (10 h).

For the May 2017 impact, the geometry was better suited. Images were acquired immediately after the impact by A.F., but showed no brightening or a darkening of the impact area. Images with some better seeing were acquired 10 min after the impact by Giancarlo Rizatto (Italy) and Philipp Salzgeberg (Austria) without any observable impact feature with a good image quality. A better observation was obtained by William Pelissard (France) $30 \mathrm{~min}$ after the impact and did not show an observable debris field. A high-resolution observation obtained $10 \mathrm{~h}$ later by Randy Christensen did not show any observable perturbation at the impact location. Finally, a methane band observation by Christopher Go $40 \mathrm{~h}$ after the impact did not show any bright feature in the planet. Figure 3 shows a selection of these images.

\section{Analysis of the impacts in March 2016 and May 2017}

\subsection{Light curves}

For each video observation, we transformed the initial video files into a sequence of numbered frames that were analyzed with a software pipeline written in IDL. The pipeline coregisters all frames by calculating the relative motions of the frames caused by the atmospheric seeing. A reference image is calculated from a stack of coregistered images and normalized by taking into account the number of frames used. The coregistration is done by an image correlation algorithm and is loosely based on the PLAYLIST pipeline for lucky imaging of planets (Mendikoa et al. 2016). The impact location is found by calculating an image built from the maximum brightness of each pixel and substracting the average brightness for each location. This generally produces an image where the impact location is well contrasted and can be found automatically. However, atmospheric seeing not only moves the planet from one frame to another, it also

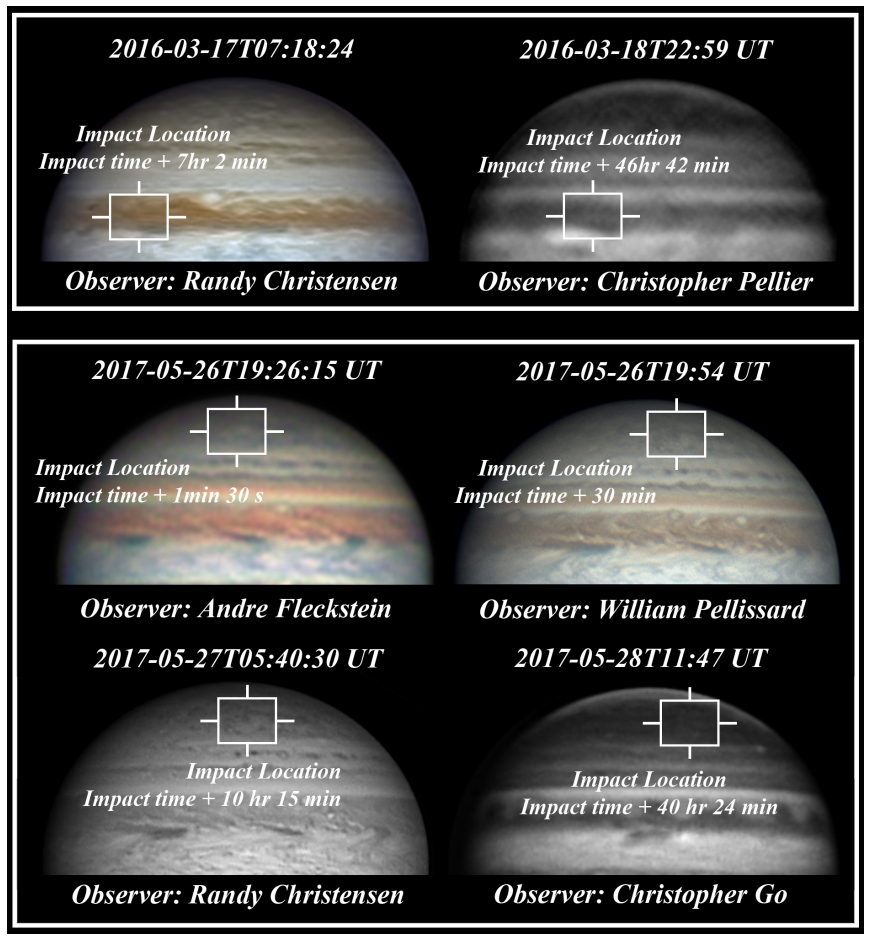

Fig. 3. Selected follow-up observations of the events in March 2016 (top panel) and May 2017 (bottom panel). In the follow-up observation by A.F., differential images with images acquired just before the flash do not show any significant difference at the impact location.

distorts the planet shape, causing the flash light to apparently move around the main impact location.

In order to calculate the light from the flash, the software calculates the difference between each frame and the reference image after coregistering each individual frame with the reference image. Differential photometry images are used to calculate aperture photometry over the impact location. Aperture photometry is done using a circular mask plus a ring to substract contributions from the background in the differential photometry images. The circular mask is recentered in each frame above the flash location by correcting distortions caused by the seeing. Additionally, the software also calculates the integrated flux of 


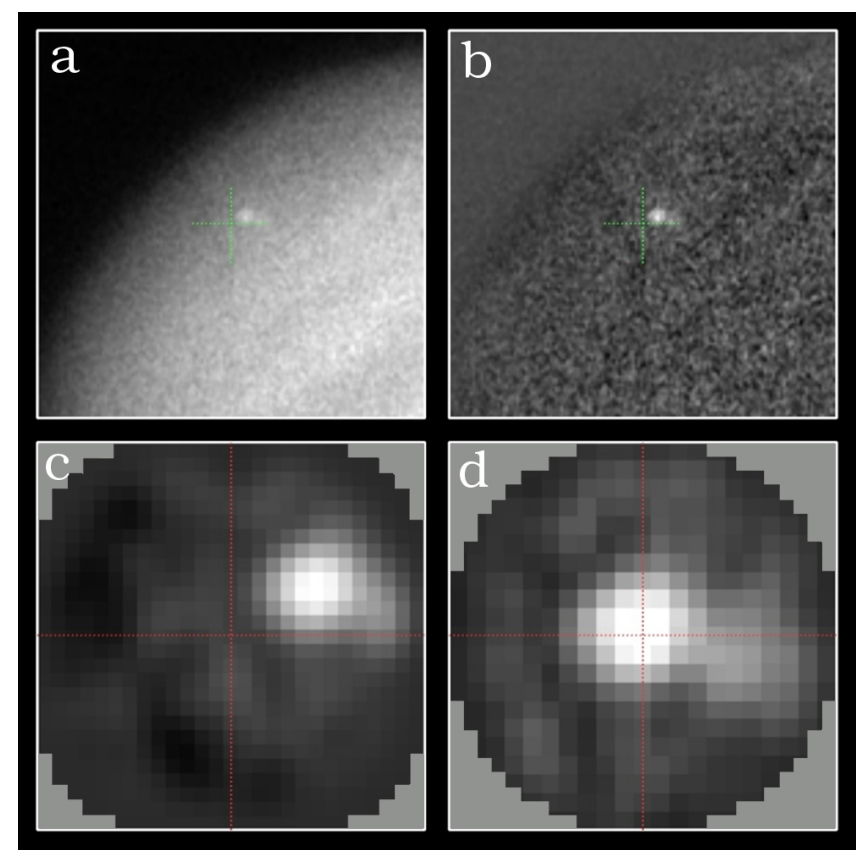

Fig. 4. Light-curve analysis pipeline. Panel $a$ : section of the original frame. Panel $b$ : differential image for that frame, i.e., the current frame minus the reference image. Panel $c$ : circular mask around the mean location of the impact. Panel $d$ : circular mask within a smaller radius containing all the light from the impact and recentered on the impact. Seeing distorts each image differently, and the location of the flash needs to be adjusted frame by frame by the software. The example is from the S.P. video observation on 2017-05-26.

Jupiter so that the brightness of the impact can be compared with the total brightness of the planet. Figure 4 shows examples of the pipeline processing. Images like these are generated by the pipeline and are used to check the correct positioning of the moving-aperture photometry mask.

For video observations with cameras that use a Bayer RGB filter, we convert the color frames into black-and-white versions using the average of all three channels. This allows better visibility of the impact and a more detailed and less noisy light-curve.

Figures 5 and 6 show raw light curves of the impacts in March 2016 and May 2017, respectively. In this case the light curve from the analysis of S.P.'s video observation shows significant temporal structure with a double central flash and an extended tail of brightness decay lasting for about $0.6 \mathrm{~s}$. The double central flash is also partially distinguishable in the second video of this event by T.R., but the smaller pixel size of the optical setup prevents us from extracting more accurate information of this video. The two flashes are readily apparent when examining the video observation frame by frame and can be related to fragmentations of the impact object. The same types of features with similar timescales are observed on light curves of superbolides on Earth (see, e.g., Fig. 3 in Borovička et al. 2017 and the extended Fig. 2 in Borovička et al. 2013). This might therefore be the first case for fragmentation of Jupiter bolides and suggests that observations of future impacts should involve a fast frame rate of at least $30 \mathrm{fps}$ to observe these characteristics. The fragmentation history of a bolide depends on the entry mass, physical nature of the meteorite, speed, and angle of the impact. A sophisticated light curve analysis can be made for Earth superbolides resulting in the physical characterization of the impactor (Borovička et al. 2017; i.e., determining the physical

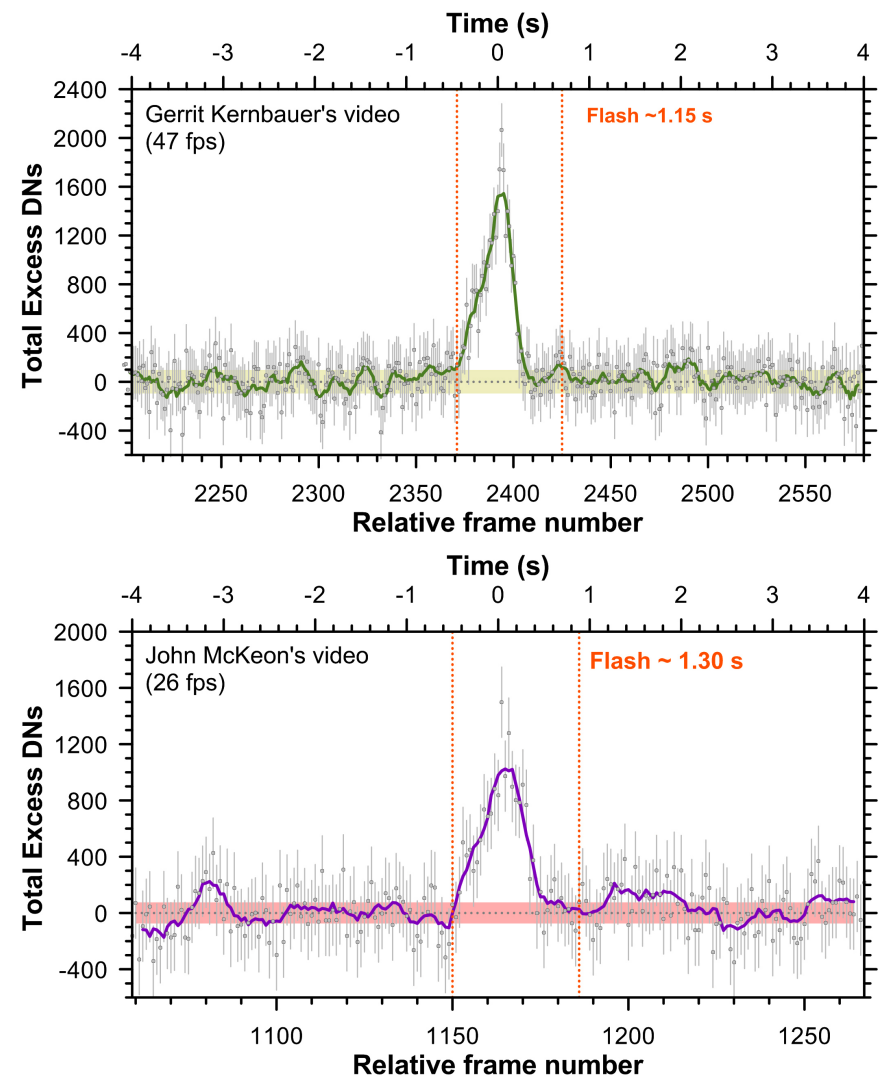

Fig. 5. Raw light curves of the impact in March 2017. Top panel: data from Gerrit Kernbauer. Bottom panel: data from John McKeon.

class of the impacting object, which can be stony, metallic, icy compact, or icy porous). The fast cameras currently used by most amateurs might start to produce such data in observations of Jupiter impacts for objects more massive than the impact in May 2017.

\subsection{Image calibration}

The total intensity from each flash, $I_{*}$, is calculated from the integrated data numbers (DNs) of each light curve. This is computed by adding the excess DNs from the minimum to the maximum times marked in Figs. 5 and 6. This number is compared with the total DNs associated with the full disk of Jupiter over the reference image built for each video, $I_{J}$. Transforming $I_{*}$ into luminous energy, $L_{*}$ (measured in Joules) is a simple problem of scaling the flash light with the Jupiter brightness as detected in each observation,

$L_{*}=K \cdot\left(\frac{I_{*}}{I_{J}}\right)$,

where $K$ is a conversion factor different for each video observation and proportional to the total flux of light reflected from Jupiter and detected with the camera.

The conversion factor was calculated in the following way: for each date, we calculated the effective solar constant at the distance of Jupiter, $S_{J}$, by scaling the solar constant at Earth, $S_{E}=1361 \mathrm{~W} \mathrm{~m}^{-2}$ to the distance of Jupiter to the Sun, $d_{J}$, using the ephemeris computed with the JPL HORIZONS system ${ }^{1}$. For each video observation we convolved the solar spectrum from

1 https://ssd.jpl.nasa.gov/ 
Colina et al. (1996) with the camera and filter response and the reflectivity spectrum of Jupiter from Karkoschka (1994) by computing the amount of energy reflected from Jupiter and detected by the camera. Because the impact photometry is computed with respect to the full disk brightness of Jupiter, the absolute values of the camera and filter response are not needed, only their relative values at different wavelengths. Thus,

$L_{*}=\left(S_{J} \cdot \pi R_{e q} R_{p}\right) \cdot\left(\frac{\int_{0}^{\infty} F_{\odot}(\lambda) \cdot I / F(\lambda) \cdot C(\lambda) \mathrm{d} \lambda}{\int_{0}^{\infty} F_{\odot}(\lambda) \mathrm{d} \lambda}\right) \cdot\left(\frac{I_{*}}{I_{J}}\right) \cdot \Delta t$,

where $R_{e q}$ and $R_{p}$ are the equatorial and polar radius of the planet, $F_{\odot}(\lambda)$ is the solar spectral radiance, $I / F(\lambda)$ is the reflectivity of Jupiter, $C(\lambda)$ is the spectral response of the camera and filter (Fig. 7 shows the spectral responses of the cameras and filters used in the detection of the two impacts), and $\Delta t$ is the exposure time for a single frame. The first term represents the flux of solar light intercepted by Jupiter. The second term is the proportion of this energy that the system can detect and contains the spectrum of Jupiter and the spectral response of the camera. The third term contains the normalization factor from the analysis of the light curve and the integrated light of the Jupiter disk. The $\Delta t$ term is used to transform Watts into Joules. The result is the "detected" luminous energy of the flash given in Joules.

The flash behaves as a punctual source of light releasing energy in all directions. Part of this light (almost 50\%) illuminates the upper clouds of Jupiter and is reflected with an average approximate albedo of 0.5 . This added contribution implies that a geometric correction needs to be introduced in the luminosity evaluation. The magnitude of the correction depends on the viewing geometry of the impact, and its exact evaluation is a complex problem of radiative transfer. For consistency with Hueso et al. (2013), this correction is here simply computed as 1.3 ,

$L_{*}^{\text {cor }} \approx L_{*} / 1.3$.

This approximate correction is applied to the impact in May 2017, but not to the impact in March 2016, which occurred too close to the planet limb, to required adding this correction from reflections of light in the Jupiter clouds.

The "detected" and corrected luminous energy $L_{*}^{\text {cor }}$ corresponds to a part of the total emitted energy in form of light. Depending on the spectral energy distribution of this light, the $L_{*}^{c o r}$ can correspond to a higher or lower amount of total emitted light. If we assume that the light is emitted at a given temperature following Planck's blackbody law, we can compute an efficiency factor for different temperatures and detectors,

$L_{*}^{f}(T)=L_{*}^{c o r}\left(\frac{\int_{0}^{\infty} F_{B B}(T, \lambda) \mathrm{d} \lambda}{\int_{0}^{\infty} F_{B B}(T, \lambda) \cdot C(\lambda) \mathrm{d} \lambda}\right)$

where $L_{*}^{f}(T)$ is the total luminous energy of the impact as a function of temperature, $T$ is temperature, and $F_{B B}$ is Planck's law of radiation for a blackbody. We considered that blackbody brightness temperatures of the flash are in the range of [3500-8500] K. These temperatures come from values of Earth's fireballs, SL9 impacts observed by the Galileo spacecraft, and analysis of the 2010 fireball on Jupiter, which was observed simultaneously at high quality with a red and blue filter (Hueso et al. 2010b). This temperature range produces a factor of two uncertainty in the
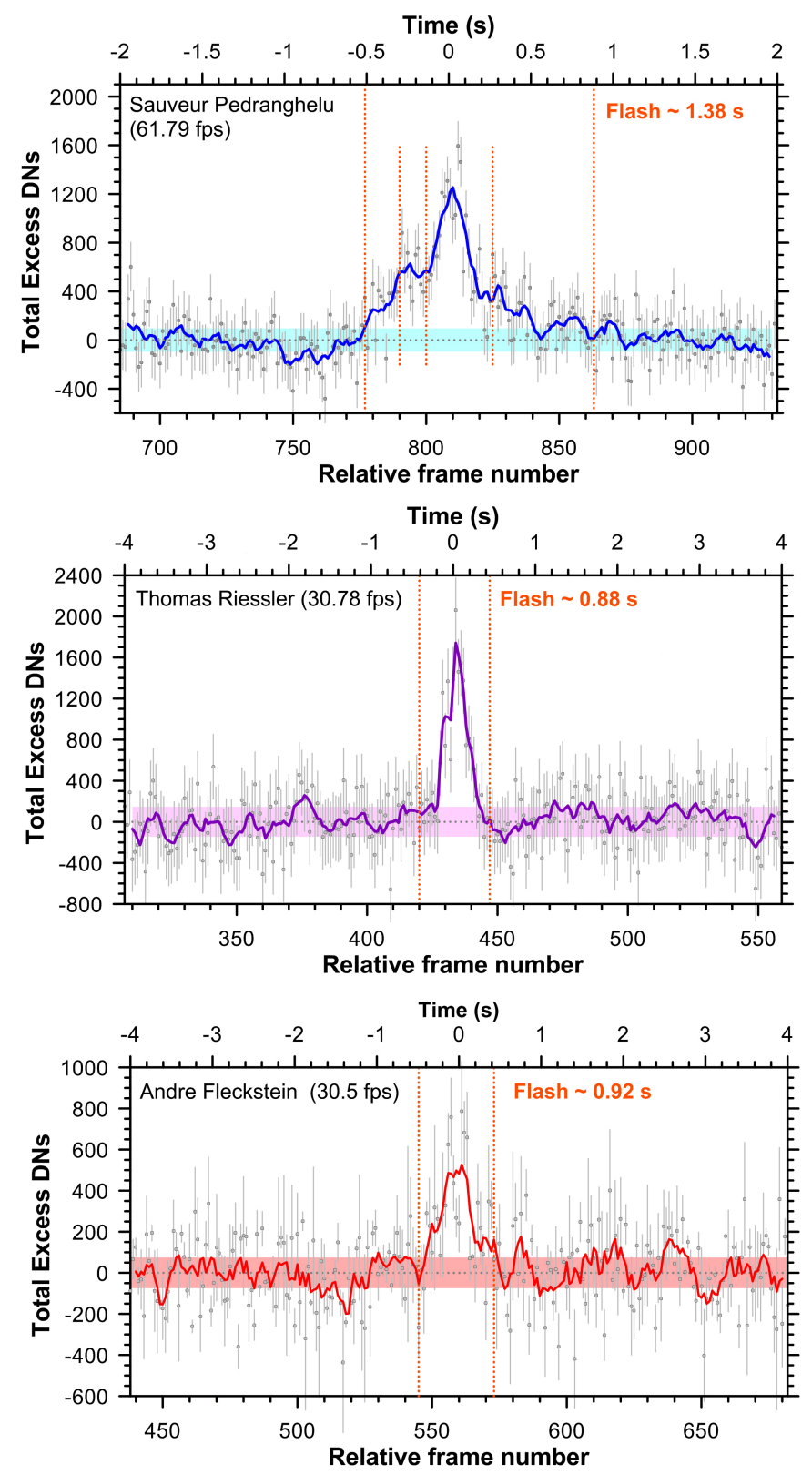

Fig. 6. Raw light curves of the May 2017 impact. Top panel: data from Sauveur Pedranghelu. The light curve has different phases that are identified with vertical orange lines: a first flash of $0.21 \mathrm{~s}$, a second phase with constant flux for $0.16 \mathrm{~s}$, a central flash of $0.40 \mathrm{~s}$, and an extended decay for another $0.32-0.61 \mathrm{~s}$ for a total flash duration of $1.38 \mathrm{~s}$. Middle panel: data from Thomas Riessler showing the double flash with a slightly shorter duration. Bottom panel: data from André Fleckstein. The video by T.R. does not show the same amount of structure visible in the first light curve, possibly because of the different frame rates and smaller pixel size. The video by A.F. was acquired under poorer seeing conditions.

energy calculation, which is larger than the uncertainties in the light-curve calculation or the geometric factor correction.

In video observations obtained with cameras that use a Bayer mask to build RGB images, we considered that the image is the sum of the three red, green, and blue channel images, so that $C(\lambda)$ is the sum of the curves representing the spectral responses of each channel.

Finally, in order to transform the total luminous energy into kinetic energy of the impactor, we need to know the efficiency 

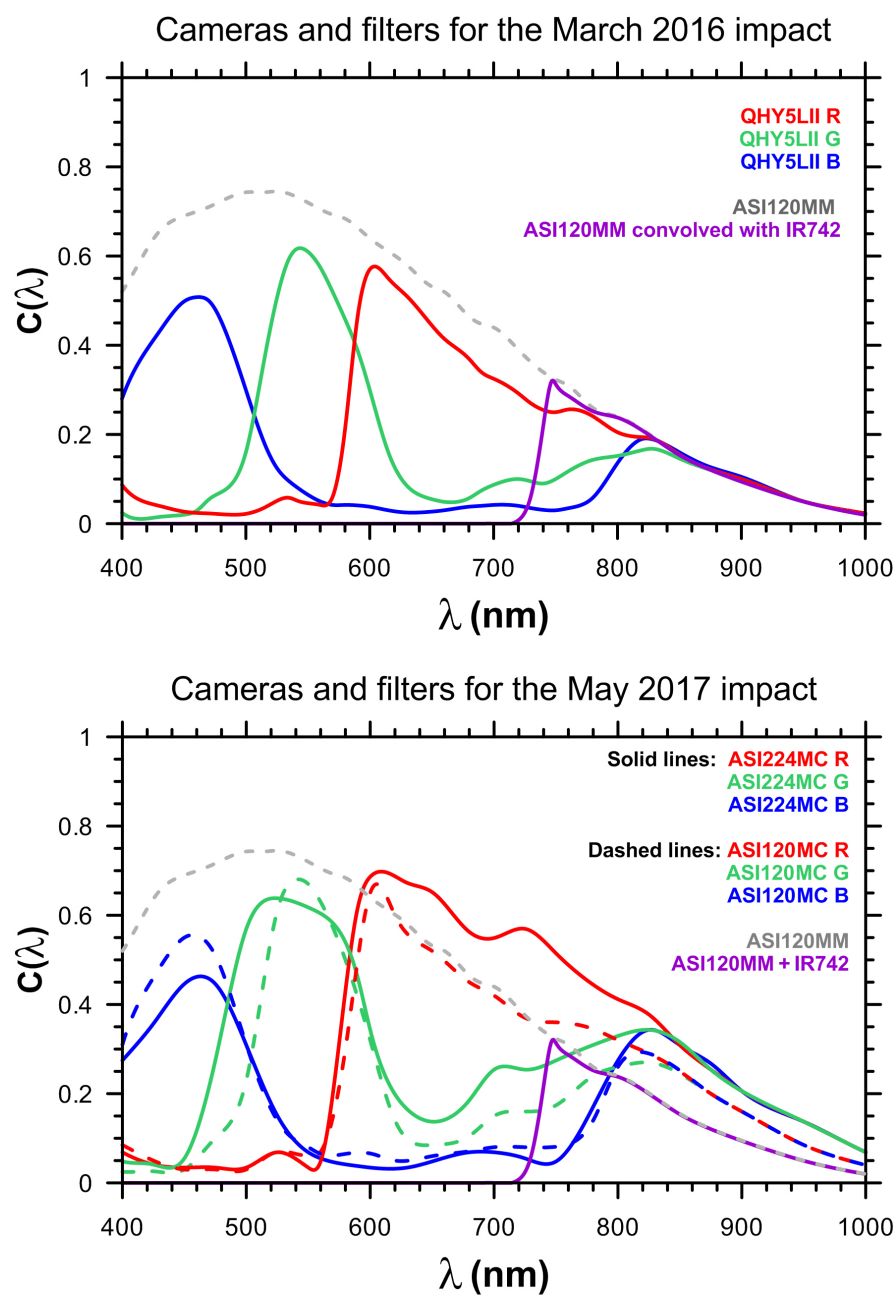

Fig. 7. Spectral responses of the combination of cameras and filters for the impacts in March 2016 (top panel) and May 2017 (bottom panel). Note the better quantum efficiency of the ASI224MC camera used by S.P. for the May 2017 impact when compared with the ASI120MC used by T.R. and the QHY5LII camera used by G.K. for the impact in March 2016. All the color filters used correspond to Bayer mask filters on the CCD.

of the impact to convert kinetic energy into luminous energy. For meteoroids and fireballs entering Earth's atmosphere, an empirical efficiency formula has been derived by Brown et al. (2002):

$\mu=0.12 E_{0}^{0.115}$

where $E_{0}$ is the optical energy measured in kilotons of TNT $\left(1 \mathrm{kton}=4.185 \times 10^{12} \mathrm{~J}\right)$.

Values of $\mu$ from this formula for Jovian impacts range from 0.15 to 0.20 . We caution that this formula is calibrated from observations of Earth impacts with optical energies from 0.001 to 1 kiloton, while Jovian impacts release optical energies in the range of 5-60 ktn. Additionally, the impacts on Jupiter occur at a different velocity with an atmosphere of a different composition. These factors introduce an additional uncertainty in the size of the impact object that is currently unconstrained.

\subsection{Masses and sizes of the impacting objects}

We assumed impacts at a velocity of $60 \mathrm{~km} \mathrm{~s}^{-1}$ close to the escape velocity of Jupiter and densities from 2.0 to $0.25 \mathrm{~g} \mathrm{~cm}^{-3}$.
The results are summarized in Table 2 in comparison with determinations of energies and masses of previous impacts. The impact in May 2017 was approximately 4.5 times less energetic than the impact in March 2016.

When examining the ensemble of impacts on Jupiter given in Table 2, kinetic energies range 32-405 ktn close to Chelyabinsklike events, which was considered to release about $450 \mathrm{ktn}$ of energy (Brown et al. 2013) and an order of magnitude lower than the Tunguska impact (5000-15000 ktn; Boslough \& Crawford 2008) or 1-3 million times lower than the combined SL9 impacts (estimated to release 300000 ktn of energy; Boslough \& Crawford 1997).

\section{Visibility of debris fields caused by intermediate-sized impacts}

The impacts we characterized have a remarkably small diversity of sizes. The largest of them was caused by an object of at most $19 \mathrm{~m}$ in diameter when considering a density of $0.25 \mathrm{~g} \mathrm{~cm}^{-3}$ similar to the assumed density for SL-9 fragments (Crawford 1997). The smallest debris field left in the atmosphere of Jupiter by one of the SL-9 fragments was caused by fragment N. This fragment was estimated to have a size of about $45 \mathrm{~m}$ in diameter (Crawford 1997). For equal density, this is about 12 times more massive than the impact flash detected in September 2012, or 180 times more massive than the smallest impact flash in May 2017. The dark debris of fragment $\mathrm{N}$ was observed in HST images before it mixed with debris from other fragments (Hammel et al. 1995).

Fragment $\mathrm{N}$ would have caused a flash 12 times brighter than the impact in September 2012, producing significant saturation over a standard impact video record (most amateurs expose each frame, so that the brightest part of Jupiter reaches about $70 \%-$ $80 \%$ of the saturation level in their detector). When we compare the estimated mass of fragment $\mathrm{N}$ and the impact in 2012, the video observation of that fragment would amount to a star of +3.3 magnitude. This is equivalent to three times the visible magnitude of Ganymede, 1/315 of the total flux of Jupiter, or $2 \times 10^{16} \mathrm{~J}$. A flash of this energy would produce a debris in the atmosphere of Jupiter within the observable reach of HST and Earth's largest telescopes. Material from the N impact and other small SL9 impacts could be observed for at least two days, but there are no reports of them a week after the impact (Spencer \& Mitton 1995).

Dissipation times of impact material in the atmosphere for previous impacts were on the order of a few months for the 2009 impact (Sánchez-Lavega et al. 2011) with an e-folding time of 10 days in the debris particles concentration (Pérez-Hoyos et al. 2012) and longer for the SL9 largest fragments (Sánchez-Lavega et al. 1998). In these impacts, the debris left in the atmosphere was spectrally dark in the continuum (with maximum contrast with the environment clouds at red wavelengths), but bright in methane absorption bands. This is so because the dark particles were deposited in the atmosphere at high altitude (pressures lower than 10 mbar; Hammel et al. 1995, 2010; de Pater et al. 2010; Pérez-Hoyos et al. 2012). The impact debris was also bright in the thermal infrared because of the heating effect of an impact and the long radiative time constant of the stratosphere of Jupiter (Harrington et al. 2004; de Pater et al. 2010).

To the best of our knowledge, the long-term visibility of a small impact debris field has not yet been explored in the literature. An empirical estimation between the debris lifetime and the size of the impactor would also depend on the nature of the 
Table 2. Jovian bolide analysis.

\begin{tabular}{lccccccc}
\hline \hline $\begin{array}{l}\text { Date } \\
(\mathrm{yr}-\mathrm{mm}-\mathrm{dd})\end{array}$ & $\begin{array}{c}\text { Optical energy } \\
(\mathrm{J})\end{array}$ & $\begin{array}{c}\text { Kinetic energy } \\
(\mathrm{J})\end{array}$ & $\begin{array}{c}\text { Energy } \\
(\mathrm{ktn})\end{array}$ & $\begin{array}{c}\text { Mass } \\
\left(10^{3} \mathrm{~kg}=\text { ton }\right)\end{array}$ & $\begin{array}{c}\text { Diameter }(\mathrm{m}) \\
\rho=2.0 \mathrm{~g} \mathrm{~cm}^{-3}\end{array}$ & $\begin{array}{c}\text { Diameter }(\mathrm{m}) \\
\rho=0.6 \mathrm{~g} \mathrm{~cm}^{-3}\end{array}$ & $\begin{array}{c}\text { Diameter }(\mathrm{m}) \\
\rho=0.25 \mathrm{~g} \mathrm{~cm}^{-3}\end{array}$ \\
\hline $2010-06-03^{a}$ & $0.3-2.5 \times 10^{14}$ & $1.9-14 \times 10^{14}$ & $46-350$ & $105-780$ & $4.7-9.1$ & $7.0-14$ & $9.3-18$ \\
$2010-08-20^{a}$ & $0.6-2.0 \times 10^{14}$ & $3.7-11 \times 10^{14}$ & $88-260$ & $205-610$ & $5.8-8.4$ & $8.7-13$ & $12-17$ \\
$2012-09-10^{a}$ & $1.6-3.2 \times 10^{14}$ & $9.0-17 \times 10^{14}$ & $215-405$ & $500-950$ & $7.8-9.7$ & $12-14$ & $15-19$ \\
$2016-03-17$ & $1.3-2.8 \times 10^{14}$ & $7.3-14 \times 10^{14}$ & $175-350$ & $403-805$ & $7.3-9.2$ & $10.9-13.7$ & $14-19$ \\
$2017-05-26$ & $1.9-3.6 \times 10^{13}$ & $1.3-2.3 \times 10^{14}$ & $32-55$ & $75-130$ & $4.1-5.0$ & $6.1-7.4$ & $8.3-10$ \\
\hline
\end{tabular}

Notes. ${ }^{(a)}$ Data from Hueso et al. (2013). Densities of $0.25 \mathrm{~g} \mathrm{~cm}^{-3}$ are considered as representative of the SL-9 impact (Crawford 1997).

impactor (stony or icy), location of the impact in the atmosphere in a region with higher or lower wind shear, and many other possible parameters (such as the impact trajectory angle with the planet). However, in the event of an impact 10 times brighter than the flashing impact that occurred in September 2010, we predict that quick follow-up observations could detect an atmospheric debris field.

We examine the detectability of impact debris on Jupiter caused by larger impacts in Sect. 7.3.

\section{Searches for new impacts}

\subsection{Dedicated detection campaigns}

Professional dedicated campaigns to detect impacts on Jupiter are difficult to carry out because such campaigns must involve the capacity of acquiring and analyzing images over many different nights. Our team runs frequent observations of Jupiter with 1-2 m size telescopes using PlanetCam UPV/EHU, a luckyimaging instrument (Sánchez-Lavega et al. 2012; Mendikoa et al. 2016). In the past five years, we have observed Jupiter over 20 different campaigns over an average of two nights per campaign, acquiring about $2.0 \mathrm{~h}$ of data for each night. These observations have been checked for impacts without observing an impact flash for an accumulated observing time of $80 \mathrm{~h}$. Details of the observations are given in Mendikoa et al. (2016, 2017).

The lack of impact detections in this survey imposes a weak constraint over the maximum impact rate on the planet. We used a simple Monte Carlo simulation to calculate the significance of this negative detection. We tested different values of the number of observable impacts per year, and for each, we launched a large-number (5000) of Monte Carlo simulations where we examined the number of impacts that would occur in $80 \mathrm{~h}$. To do this, we divided $80 \mathrm{~h}$ into 4800 opportunities of $1 \mathrm{~min}$ to detect an impact. We examined the statistics of the Monte Carlo simulation and searched for the number of observable impacts per year that result in $66 \%$ of the simulations producing at least one impact in an accumulated observing time of $80 \mathrm{~h}$. The statistical result is that an impact rate of 120 detectable impacts per year would be needed to find one impact in this observing time. The Monte Carlo simulation shows that for a probability of $90 \%$ to find an impact in a survey of $80 \mathrm{~h}$, we would require an impact rate of 250 observable impacts per year. This analysis suggests an upper limit on the impact rate smaller than 120 impacts per year.

A project run by Japanese amateurs called "Find Flash" and run by the Association of Lunar and Planetary Observers (ALPO) in Japan with more than 50 amateur observers and about 10 nights per year observation time on $1 \mathrm{~m}$ size telescopes did not find impacts for about four years of observations, placing a similar constraint (I. Tabe, priv. comm.).

\subsection{Filters and technology}

Flashes can be best detected in filters where the planet is dark and the integration time of the camera is not too long. Blue filters and relatively wide filters centered on the methane absorption band at $890 \mathrm{~nm}$ are best suited for a flash detection because the planet is dark and the flash should be bright. Integration times lower than $0.2 \mathrm{~s}$ are needed, suggesting that while blue filters can be used with small telescopes, an instrument with a minimum diameter of $30 \mathrm{~cm}$ might be required to perform a flash detection campaign for the $890 \mathrm{~nm}$ methane band. Dual observations in blue and $890 \mathrm{~nm}$ would highly constrain the brightness temperature of the flash, allowing for a determination of the impact energy with lower ambiguities.

The cameras used in the amateur community have experienced significant improvements over the past decade from read noises of $\sim 8 \mathrm{e}-$ at 0 gain (e.g., in the popular Point Grey Flea3 camera used for the first detections of impacts in Jupiter) to $\sim 3 \mathrm{e}-$ at 0 gain (in the ASI cameras now used by most amateurs). Quantum efficiencies have improved at least by a factor of two in the near-infrared, where most observers concentrate their observational efforts, and have increased from 5\% to $30 \%$ in the $900 \mathrm{~nm}$ range. Better sensitivity results in impacts being detectable by smaller telescopes with faster exposures and better temporal resolution of the light curves.

\subsection{Impacts DeTeCtion software}

We have written a software tool to inspect amateur video observations of Jupiter capable of detecting impacts in the planet. The latest version of this software, DeTeCt3.1, is an open-source application that amateur observers can use on their own computers (Delcroix et al. 2017). The software constitutes one of the activities of the "Planetary Space Weather Services" (PSWS; André et al. 2018) and is based on differential photometry of coregistered images. This software is essentially different from "flash detection" software developed for search of impact flashes on the Moon (Madiedo et al. 2015) because these tools generally examine videos acquired over a large field of view (almost the full non-illuminated side of the Moon) without the need to correct for seeing effects (coregistration) and because the flash is recorded over a dark object and not the bright background of Jupiter.

The DeTeCt software has been used regularly by dozens of observers examining about 70000 video files, which is equivalent to more than 75 days of observations distributed unevenly over several years. The software produces log files that are later analyzed to examine the statistics of nondetections when comparing with the fortuitous detections of impacts. The analysis shows that about $5 \%$ of all observations were acquired at the 


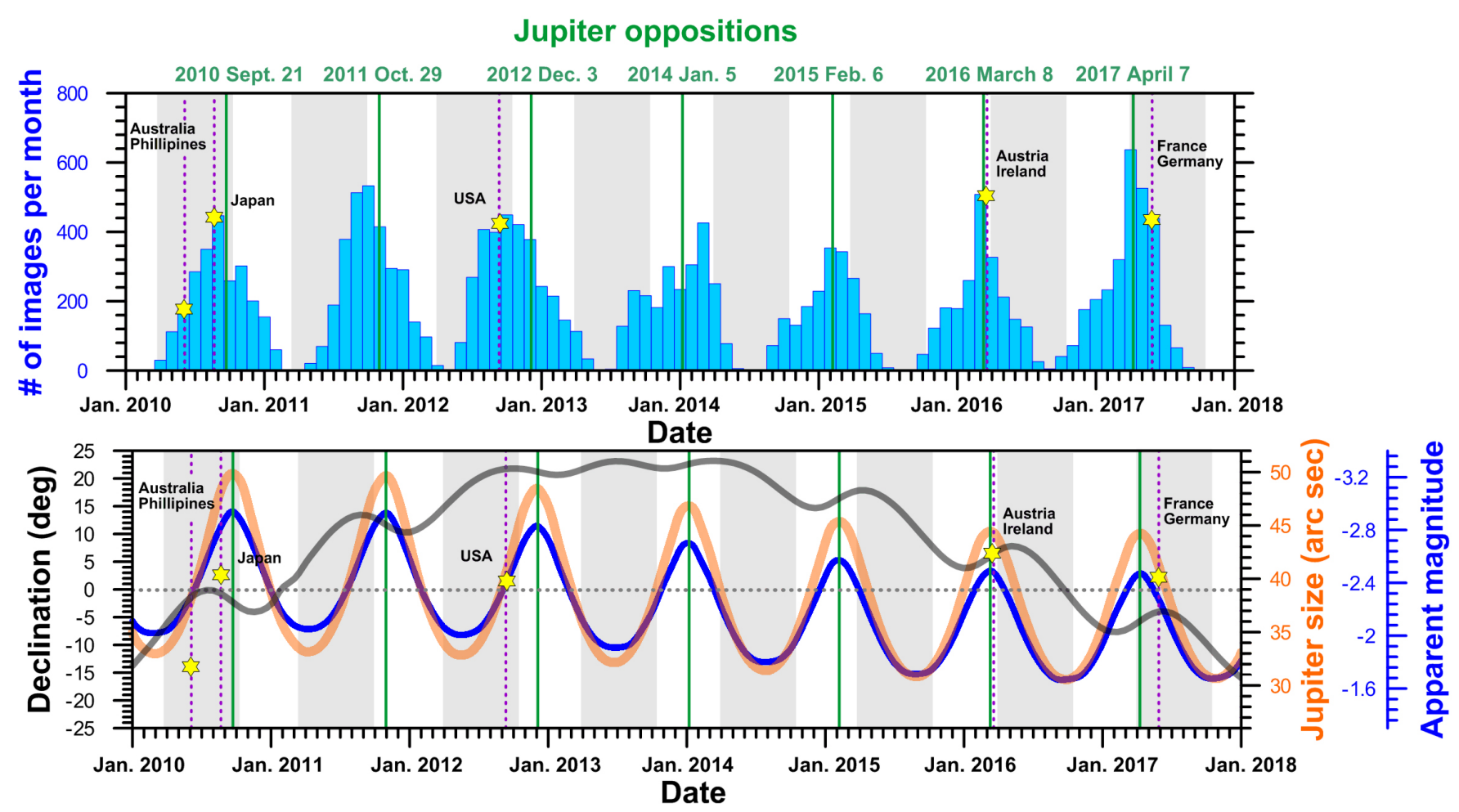

Fig. 8. Number of Jupiter images per month archived in the PVOL database (top panel) and observation conditions (bottom panel) as a function of time. Jupiter oppositions are shown with solid green lines and are labeled on the uppermost horizontal axis. Dates of Jupiter impacts are shown with vertical dotted magenta lines and yellow stars. The region where the impact was observed labels each line. Spring and summer months in the north hemisphere are shown in gray, and winter and fall in the north hemisphere are shown in white. Bottom panel: Jupiter declination (left axis and black line) compared with the planet apparent size in arcsec (right axis and orange line) compared with its visual magnitude (blue line and axis). The horizontal dotted gray line shows the zero-declination line.

same time by the collaborating observers. The software and statistical analysis of its results can be accessed online ${ }^{2}$. A similar analysis of video observations of Saturn is also available at that web site, with negative results so far.

From the statistics of the time covered by these observations, and because one of the observers of impacts in Jupiter (J. McKeon) uses this software regularly, one can consider a detectable impacts rate of $1 / 75$ days $^{-1}$ ), which is equivalent to 4.9 observable impacts per year. This is a minimum number because most video files are acquired with regular sky conditions, and we cannot measure the efficiency of finding impacts on low-quality video files.

\section{Statistical analysis of the impacts}

\subsection{Continuous flux or meteor showers?}

Several works have predicted the existence of meteor showers on other planets based on the characteristics of known comet/meteoroid orbits that cross the nodal points of planets. Most of them considered only the inner planets (e.g., Christou 2010), but Selsis et al. (2004) presented a study for all solar system planets, including Jupiter and Saturn. They found 48 "comet candidates" that could produce meteor showers on Jupiter. In contrast to what happens on the inner planets, meteor showers in Jupiter largely overlap in time because of the very long duration of close comet passages. We therefore assume that impacts of the class detected by amateur astronomers should

\footnotetext{
2 http://www.astrosurf.com/planetessaf/doc/project_ detect.shtml
}

be distributed randomly in time and not be clustered when the Jupiter orbit traverses a cometary tail. We also assume that these impacts occur close to the escape velocity of Jupiter of $60 \mathrm{~km} \mathrm{~s}^{-1}$, in agreement with observations of the SL9 impact (Harrington et al. 2004).

\subsection{Temporal survey of Jupiter from amateur observers}

Understanding the significance of the five flashes requires knowledge about when the amateur community performs their observations of Jupiter. Figure 8 shows a statistical analysis of Jupiter observations archived at the PVOL database (Hueso et al. 2010b, 2018). This is one of the most popular databases of amateur observations of solar system planets and can be searched with very many parameters. In the period from January 2010 to December 2017, the PVOL database contains 17643 Jupiter observations that are representative of global trends in amateur observations of Jupiter. Each year, the observations cluster more abundantly close to the opposition of Jupiter. In years where this opposition is close to winter in the north hemisphere, fewer amateurs are able to observe the planet regularly. This is due to the geographical distribution of most amateur observers. About $65 \%$ of all Jupiter observations come from observers in the north hemisphere, with about $21 \%$ of observations contributed from $\pm 30^{\circ}$ latitudes and $16 \%$ from south hemisphere latitudes. The number of Jupiter observations for the 2010-2011 Jupiter apparition was about 2400 . This number reduced by about $25 \%$ in the three Jupiter apparitions in 2013-2015 and increased by about 15\% in the latest 2016 and 2017 Jupiter apparitions.

This trend with Jupiter oppositions helps to explain the gap in the detection of Jupiter flashes in the period 2013-2015 when 
Jupiter opposition resulted in most observers having difficulties to find good weather and with generally fewer observations (Fig. 8). Jupiter oppositions in 2018-2022 will occur from May to September, offering increased capabilities of detecting impacts.

\subsection{Statistical interpretation of the flashes}

It is difficult to make statistical analysis of events that have been observed only a few times. The results in Sect. 5 provide absolute upper limits and weak lower limits to the observable impacts on Jupiter. Here we present different arguments to infer the number of detectable impacts on Jupiter.

1. An absolute minimum flux of impacts on Jupiter of 0.63 impacts per year is found based on the five flashes detected in eight years (2010-2017).

2. The statistical analysis from DeTeCt can also be understood as a minimum flux of 4.9 impacts per year. Only one of the 11 observers that have successfully found an impact on Jupiter collaborates with this project regularly. If this fraction is representative of impacts on the planet, then the 4.3 impacts per year could scale up to 52 impacts per year.

3. Hueso et al. (2013) gave order-of-magnitude estimates considering the geographical distribution of observers and the number of observations per year of the amateur community, inferring between 6 and 30 detectable impacts per year based on the three impacts detected from 2010 to 2012. We here correct these estimates with an update of the number of impacts detected in the period 2010-2017. We assume that the total surveyed time is given by

$T=N \cdot t_{1} \cdot e$,

where $N$ is the total number of images, 17643 as stated above, $t_{1}$ is the time accumulated to form each image, and $e$ is the efficiency for each image to have enough quality to show an impact. $t_{1}$ can be from 5 to $15 \mathrm{~min}$ since each image is the result of a longer observing session. The efficiency $e$ in which a video observation can have enough quality so as to show an impact was estimated to be from 0.3 to 0.5 in Hueso et al. (2013). Then $T$ is approximately 18-91 days over an accumulated time of eight years. Only 2 of the 11 observers who have found impacts in Jupiter are regular contributors to this database, and we estimate that the global network of amateur astronomers can be represented by an increase in Jupiter observing time by a factor of 11/2. Therefore, we consider that the global survey of Jupiter observations by the amateur community can be globally represented by a total observation time of 99-500 days obtained over the past eight years. This represents a global observation efficiency of Jupiter of 3.4-17\%. In this way, the five impacts detected in eight years may scale up to an estimate of 4-18 "detectable" impacts per year. Even if we were to consider the amateur observations as a "perfect survey" with a detection efficiency of $e=1.0$, the detectability of impacts would still be limited by the geographical distribution of observers clustered in North and South America, Europe, and Japan-Australia. This would result in an observing time efficiency of $33 \%$ of the total available time and a minimum number of 2.5 detectable impacts per year.

4. Of the 11 observers that have detected impacts in Jupiter, 2 can be considered as very regular observers performing an outstanding number of Jupiter observations every year and participating in several research projects (A.W. and C.G.). One of them (A.W.) discovered the debris of an impact in 2009 (Sánchez-Lavega et al. 2010) and the first flash of light the next year. A.W. accumulates $180 \mathrm{~h}$ of Jupiter observations per year, personally looking at every video, and half of these data have a quality good enough to visualize a small impact ( $90 \mathrm{~h}$ per year). This means that he has found one (two) impact(s) in an accumulated observing time of 720 (810) h over the past eight (nine) years, where the number in parentheses indicate whether we also consider his initial finding of the 2009 large impact. For A.W. alone, this is about $35-40 \%$ of all the time covered by the DeTeCt program. This suggests detectable impacts with a rate close to 10-20 impacts per year.

5. The second impact on Jupiter was found only two months after the first. The clustering of these two events close in time can be examined with Monte Carlo simulations. We simulated impacts considering different impact frequencies in a Monte Carlo simulation representative of eight years, where for each day, we calculated the random probability of having found an impact. Detection was examined considering (i) that each day, the planet could only be observed $33 \%$ of the time because of the longitudinal distribution of Earth observers that peaks over Europe, North America, and Japan meridians; (ii) that observations only cover nine months of a year; (iii) that detecting the impact was not possible because of poor weather $50 \%$ of the time; and (iv) that detections were not possible because of poor atmospheric seeing $50 \%$ of the time. This renders the detection probability of any given impact as $6.3 \%$. Two impacts occur with a time difference shorter than three months in about $50 \%$ of the Monte Carlo simulations, with an impact flux of 5-15 impacts per year.

All in all, we consider that a reasonable estimate of the number of potentially detectable impacts per year in Jupiter from objects of 5-20 m size or larger can be on the order of 4-25. However, since we only observe the planet nine months every year and we can only observe about one half of its surface at any given time, the "detectable" number of impacts corresponds to a higher number of objects colliding with Jupiter. This correction means that the accumulated flux of objects larger than 5-20 m in diameter that hit Jupiter every year is estimated to be 10-65, compared with 12-60 from Hueso et al. (2013).

\section{Impact flux on Jupiter}

\subsection{Consequences for the chemical composition and dust abundance in the Jovian stratosphere}

Based on the flux rate derived in the previous section, the contribution of impacts of this size range to the delivery of chemical species and dust to the upper atmosphere of Jupiter is expected to be on the order of $8 \times 10^{5}-7 \times 10^{7} \mathrm{~kg} \mathrm{yr}^{-1}$. Recent research on Earth large-size bolides shows that the largest fraction of the impacting object is deposited in the atmosphere in the form of micrometer dust (Klekociuk et al. 2005). This contribution to exogenous species and dust can be compared with the continuous contribution from interplanetary dust particles (IDPs) colliding with Jupiter. Current models of interplanetary dust fluxes on the giant planets (Poppe 2016) predict about $10^{-13} \mathrm{~g} \mathrm{~m}^{-2} \mathrm{~s}^{-1}$ or $1.9 \times 10^{9} \mathrm{~kg} \mathrm{yr}^{-1}$. The dust observations made by the Galileo Dust Detection System (DDS) of impact-induced ejecta clouds around the Galilean Moons (Krüger et al. 1998, 2000, 2003) result in estimates of the total mass flux of IDPs on Jupiter of $3 \times 10^{-13} \mathrm{~g} \mathrm{~m}^{-2} \mathrm{~s}^{-1}$ (Sremčević et al. 2005), which is equivalent to $5.6 \times 10^{9} \mathrm{~kg} \mathrm{yr}^{-1}$. However, these estimates are probably uncertain by an order of magnitude (Poppe 2016). Thus, the contribution of impacts of the size range such as those discovered in observations of Jupiter fireballs contribute about $0.01-3.7 \%$ of the IDP exogenous material and dust to the upper stratosphere 
R. Hueso et al.: Small impacts on the giant planet Jupiter

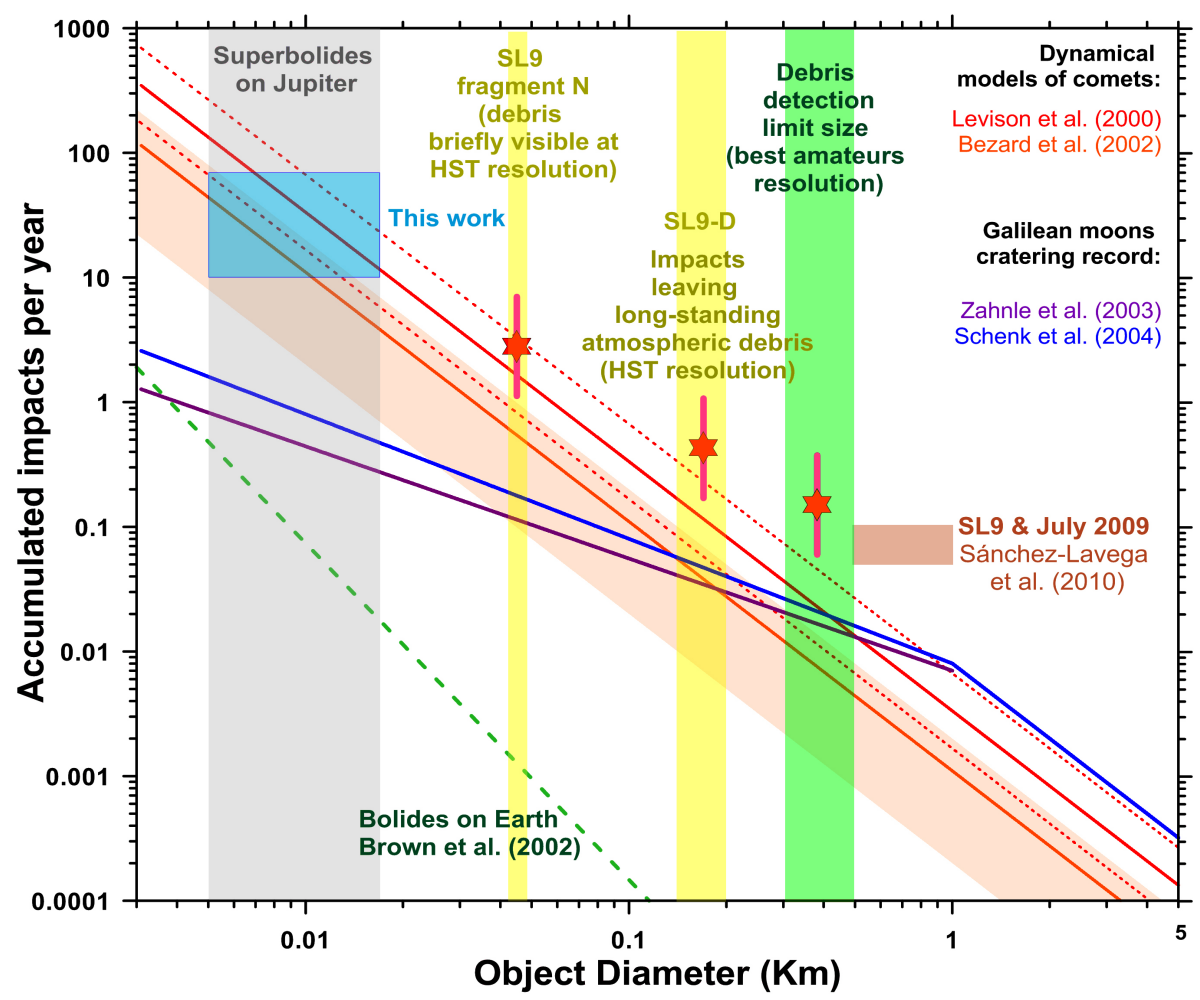

Fig. 9. Impact rates on Jupiter and Earth compared. The vertical gray region represents the sizes of the five bolides, and the blue box represents the impact rate of 5-20 m size objects on Jupiter. The lines represent impact rates from (a) dynamical models of comets (red line with estimated uncertainties as the dotted line, Levison et al. 2000; (b) orange line and estimated uncertainties from corrections to that model introduced by Bézard et al. 2002, or; (c) the cratering record of Galilean moons (magenta line from Zahnle et al. 2003; blue line from Schenk et al. 2004). This is compared with estimates of the impact rate on Earth from Brown et al. 2002). The yellow regions indicate the limiting size of objects that might be discovered based on the dark debris that they are expected to leave in the atmosphere of Jupiter: The smallest SL9 fragment that produced a detectable debris field on HST images (fragment N) and long-standing debris features associated with objects of $170 \mathrm{~m}$ such as SL9 fragment D are highlighted. The green vertical region represents objects leaving a debris field that might be detected with amateur equipment. The light-brown box represents estimates of impact rates on Jupiter from Sánchez-Lavega et al. (2010) for $0.5-1.5 \mathrm{~km}$ size objects. Estimates of the impact rate of intermediate-size objects that hit the planet and form observable debris fields are plotted as red stars. The error bars represent an uncertainty factor of 2.5 upward and downward. Figure updated from Hueso et al. (2013).

of Jupiter and are not expected to have a strong impact even on local scales.

Lellouch et al. (2002) have analyzed ISO observations and found an upper limit $\left(8 \times 10^{4} \mathrm{~cm}^{-2} \mathrm{~s}^{-1}\right)$ to the permanent water influx into the stratosphere of Jupiter. Bézard et al. (2002) have placed oxygen influx limits at $(1.5-10) \times 10^{6} \mathrm{~cm}^{-2} \mathrm{~s}^{-1}$ based on observations of $\mathrm{CO}$. Various possibilities for the high exogenous $\mathrm{CO} / \mathrm{H}_{2} \mathrm{O}$ ratio at Jupiter have been considered by Bézard et al. (2002) and are further discussed by Poppe (2016) and Moses \& Poppe (2017). The main conclusion is that favorable production of $\mathrm{CO}$ over $\mathrm{H}_{2} \mathrm{OH}_{2} \mathrm{O}$ during IDP ablation is needed to explain this result. In the case of the contribution of 5-20 m size impacts, the same conclusions apply, that is to say that the incoming water in impacts has to be transformed into $\mathrm{CO}$. The water influx of $8 \times$ $10^{4} \mathrm{~cm}^{-2} \mathrm{~s}^{-1}$ from Lellouch et al. (2002) is equivalent to a flux of $143000 \mathrm{~kg}$ of water per year. This amount of water could be supplied by a single impact of $10 \mathrm{~m}$ with $30 \%$ of water if water could be preserved during the impact. We conclude that the water molecules ablating from the incoming impacting object must be thermochemically converted into $\mathrm{CO}$ soon after ablation.

\subsection{Impact flux as a function of impactor size}

Table 3 summarizes the masses of all impacts observed on Jupiter from the SL9 series of impacts down to a very small meteor entering the atmosphere of Jupiter that was observed
Table 3. Summary of impacts on Jupiter.

\begin{tabular}{lll}
\hline \hline Date & Mass & Reference \\
\hline $\begin{array}{l}1981-03-05 \\
1994-07-16 \text { to }\end{array}$ & $11 \mathrm{~kg}$ & Cook \& Duxbury (1981) \\
1994-07-24 & & Hammel et al. (1995) \\
$2009-07-19$ & $6.0 \times 10^{7} \mathrm{Tn}$ & Sarrington et al. (2004) \\
$2010-06-03$ & $105-780 \mathrm{Tn}$ & Hueso et al. (2010b, 2013) \\
$2010-08-20$ & 205-610 Tn & Hueso et al. (2013) \\
$2012-09-10$ & 500-950 Tn & Hueso et al. (2013) \\
$2016-03-17$ & 403-805 Tn & This work \\
$2017-05-26$ & $75-130 \mathrm{Tn}$ & This work \\
\hline
\end{tabular}

by Voyager 1 (Cook \& Duxbury 1981). Other plausible but unconfirmed collisions with Jupiter have been proposed, such as a dark spot on Jupiter observed by Cassini in 1690 with morphological characteristics and behavior similar to SL9 debris features (Tabe et al. 1997) that were proposed to be caused by an impact with a $600 \mathrm{~m}$ object (Zahnle et al. 2003).

Figure 9 presents an update of results presented in Hueso et al. (2013) of our understanding of the current flux of impacts on Jupiter. The results from this work are very similar to our previous analysis. The deduced impact rate for 5-20 m size objects compared with estimates of greater impacts from 
Table 4. Predictions of impacts per year on Jupiter that leave observable debris fields, and their detectability.

\begin{tabular}{lcccc}
\hline \hline Size $(\mathrm{m})$ & $\begin{array}{c}\text { Mean } \\
\left(\mathrm{yr}^{-1}\right)\end{array}$ & $\begin{array}{c}\text { Min } \\
\left(\mathrm{yr}^{-1}\right)\end{array}$ & $\begin{array}{c}\text { Max } \\
\left(\mathrm{yr}^{-1}\right)\end{array}$ & $\begin{array}{c}\text { Detectability } \\
(\mathrm{yr})\end{array}$ \\
\hline 45 & 2.8 & 1.1 & 7.0 & $0.4-2.6$ \\
170 & 0.43 & 0.17 & 1.1 & $2-12$ \\
380 & 0.15 & 0.06 & 0.37 & $6-30$ \\
\hline
\end{tabular}

Notes.The impact frequency is the number of impacts that we estimate to occur on Jupiter every year. Their detectability (the mean number of years between observable impacts of a given size and larger) is affected by observational biases, as discussed in detail in Sect. 7.3.

Sánchez-Lavega et al. (2010) can be interpolated to predict plausible impact rates of intermediate-size objects, as shown with stars in Fig. 9. Error bars here represent uncertainties of 2.5 higher or lower from the overall uncertainty of the impact rate of small-size impacts (blue box in Fig. 9). This is compared with expectations from dynamical models of comets prone to Jupiter collisions (Levison et al. 2000) and the cratering record on the Galilean moons (Zahnle et al. 2003; Schenk et al. 2004). Predictions of impact rates from this study are comparable to the upper estimates of impact rates from Levison et al. (2000). Our results for small-size impacts clearly depart from impact rates estimated from cratering of the Jovian moons, which for small impacts are dominated by young craters on Europa (Schenk et al. 2004).

\subsection{Searches of debris left by impacts}

We now focus on predictions based on Fig. 9 of the plausible impact rate of larger objects that might be detected as an intense flash and might leave an observable trace in the atmosphere. The detectability of these impacts depends not only on the impact frequency in Fig. 9, it also depends on their size and the biases associated with their detection. Predicted impact rates in the planet and their detectability are summarized in Table 4 and are discussed below.

Large superbolides. Voyager and Cassini observed Jupiter at high resolution over a period of at least three months. For Cassini, a global coverage at spatial resolutions of about $140 \mathrm{~km} /$ pix or better was acquired for at least 15 days (Porco et al. 2003; Salyk et al. 2006). Objects of 5-20 m hitting the planet with the flux rate deduced from this work would give a non-negligible probability (0.5-3 impacts over 15 days) to have occurred in the course of this Cassini 15-day window. Higher resolutions over particular regions were acquired for another 30 days. Thus, small debris fields in the methane band and ultraviolet images where the debris maximizes its contrast might exist in the Cassini imaging data. On Earth, many satellites have observed high-atmosphere debris associated with impacts. The best case are the satellite observations of the Chelyabinsk impact, which was observed at a variety of spatial resolutions $1-10 \mathrm{~km} \mathrm{pix}^{-1}$ for at least $3 \mathrm{~h}$ (Miller et al. 2013). This impact was similar in energy to the Jupiter impacts we discussed here. Although other missions have imaged Jupiter (Pioneers, New Horizons, and Juno), the number of images from these missions is too low to merit a specific analysis, but for Cassini and the Voyagers, a search for tiny- and small-debris fields might place an important constraint in the rate of impacts on the planet.

Small impacts. Based on this analysis, objects of $45 \mathrm{~m}$ or larger that leave a short-lived debris field that is only observable with large telescopes may impact Jupiter once every 0.36 years with uncertainties from 0.14 to 0.9 years. Since they can impact on the far side of the planet or in months when Jupiter is not observable, even a perfect survey of impacts on Jupiter could only find these events once per year with estimated uncertainties from 0.4 to 2.6 years. HST observations of the fragment $\mathrm{N}$ impact site did not allow determining for how long debris from this impact might be observable. The visibility of such an impact may also depend on its latitudinal location and on dissipation effects such as local wind shear. A careful examination of archived HST images of Jupiter acquired since 1991 (the date of the first Jupiter observation) and a search for tiny dark spots in the visible or that are bright in methane may place additional constraints on the impact rate on Jupiter or serve to lower the impact rate deduced from small-size flashing impacts. This is a non-trivial effort because archived HST Jupiter images encompass more than 350 target names, several instruments, tens of filters, and observing programs that range from global coverage to snapshots, and the debris field might be within the limit of detectability in most filters. Additionally, small impact debris fields would not be observable in subpolar latitudes.

Intermediate-size impacts. Objects larger than $170 \mathrm{~m}$ that leave a debris field that might be observable with small telescopes over weeks and months with professional telescopes may occur once every 2.3 years with estimated uncertainties from 1 to 6 years. Their detectability is difficult to ascertain because these events might be observed in only about half of each year when Jupiter is well placed in the night sky for astronomical observation. Therefore a timescale of 2-12 years seems reasonable for the detectability of these events.

Large impacts. Objects larger than $380 \mathrm{~m}$ that are able to leave a debris field that is observable in standard amateur images over weeks and during months in observations with professional telescopes may only occur once every 7 years with uncertainties from 3 to 16 years. Again, since Jupiter is only observable nine months every year and the quality of observations is a function of the proximity to Jupiter opposition, these events may be discovered by amateurs about once every 6-30 years, similarly to the timescale separation between the SL-9 collision and the 2009 impact.

\section{Conclusions}

The most recent two impacts on Jupiter in March 2016 and May 2017 had masses of 310-620 and 75-130 Tn, respectively, with sizes ranging from $4.1 \mathrm{~m}$ to $17 \mathrm{~m}$ for object densities from 2.0 to $0.25 \mathrm{~g} \mathrm{~cm}^{-3}$. These masses are comparable to previous impacts on Jupiter that have also been found in flashes in video observations of the planet.

The cumulative impact rate of objects of this size range or larger is predicted to lie in the range of 10-65 per year, with only 4-25 impacts per year being observable in a perfect survey of flashes because of the way they are distributed over Jupiter's visible and far sides and the Jupiter observation period per year.

The overall impact rate for Jupiter according to this work is similar to the high impact rate limit of Levison et al. (2000) and compatible with the modifications discussed by Bézard et al. (2002). Although significant uncertainties exist, the observations of impact flashes on Jupiter disprove the low impact rate predicted from the cratering record on Europa that was discussed by Schenk et al. (2004). 
Five to twenty meters size objects impacting Jupiter are expected to be detected yearly in the next Jupiter oppositions because of the improved observing conditions and the availability of software tools.

Future observations of impacts with the modern cameras currently available to the amateur community could be obtained at $60 \mathrm{fps}$ or higher with a good signal-to-noise ratio. For an energetic impact like those of 2012 and 2016, this may allow exploring the fragmentation history of impacting objects, opening the possibility of studying the nature of the impacting object (stony, metallic, icy compact, or icy porous).

The accumulated effect of these impacts on the chemistry of the upper stratosphere of the planet is negligible when compared with other sources of exogenous chemicals, such as interplanetary dust particles and giant impacts.

A "large flash" comparable to fragment N of SL9 leaving an observable debris field at the limit of spatial resolution with HST, VLT, or other large telescopes might occur on Jupiter with a typical timescale of once every 2-11 months. A perfect observational survey of bright flashes would find these powerful flashes about once per year. A dedicated careful examination of all HST observations of Jupiter obtained since 1991 that would search for small dark spots on visible images and bright spots on methane images might help to reduce the factor of 6 uncertainty on the impact rate from this work. A similar search for smaller impact debris from Voyager and Cassini images seems worthwhile, based on this study.

An extremely intense flash leaving a standing debris field in the atmosphere of Jupiter that could be observed with HST or large ground-based telescopes over weeks might occur on Jupiter once every 1-6 years. Regular ground-based observations of debris fields on Jupiter might detect these events about once every 2-12 years and more efficiently than a survey of flashes.

Impacts leaving a debris field that would be observable with amateur equipment might occur on Jupiter once every 3-16 years and might be observable once every $6-30$ years when accounting for the time of the year when Jupiter can be observed at high resolution by amateur astronomers.

Future observations will find increasingly smaller impacts as the technology improves. Specific searches in Voyager, Cassini, and HST images may contain small-debris fields that were not detected at the time of the acquisition of these observations. If these "small impact scars" are detected, they will largely constrain the impact rate on Jupiter. The JUICE mission to Jupiter may also discover flashing impacts on the planet that are caused by objects of much smaller sizes through the long surveys of the Jupiter night side that are currently planned for studies of the Jovian magnetosphere and the deep lightening activity (Grasset et al. 2013).

Acknowledgements. We are very grateful to Thomas Riessler for permission to work on his recording of the May 2017 impact. We are also grateful to Emmanuel Lellouch for a detailed and constructive review of this research. We thank C. Go, M. Tachikawa, K. Aoki, M. Ishimaru, D. Petersen, and G. Hall from their observation of impacts in Jupiter as well as I. Tabe for help in communicating with Japanese amateur astronomers. We also thank Sebastian Voltmer for providing early information from Gerrit Kernbauer's video detection and image processing of this video, and Dan Fischer for his help in finding German-speaking observers that had observed the May 2017 impact. We are also very grateful to the ensemble of amateur astronomers running $\mathrm{DeTeCt}$ on their video observations of Jupiter, and to the large community of observers providing Jupiter observation data through PVOL and ALPO Japan. R.H. and A.S.L. were supported by the Spanish project AYA2015-65041 (MINECO/FEDER, UE), Grupos Gobierno Vasco IT-765-13 and UPV/EHU UFI11/55. This work has been supported by the Europlanet 2020 Research Infrastructure. Europlanet 2020 RI has received funding from the European Union's Horizon 2020 research and innovation programme under grant agreement No 654208.

\section{References}

André, N., Grande, M., Achilleos, N., et al. 2018, Planet. Space Sci., 150, 50 Bézard, B., Lellouch, E., Strobel, D., Maillard, J.-P., \& Drossart, P. 2002, Icarus, 159,95

Borovička, J., Spurný, P., Brown, P., et al. 2013, Nature, 503, 235

Borovička, J., Spurný, P., Grigore, V. I., \& Svoreň, J. 2017, Planet. Space Sci., 143,147

Boslough, M. B. E., \& Crawford, D. A. 1997, Ann. N. Y. Acad. Sci., 822, 236

Boslough, M. B. E., \& Crawford, D. A. 2008, Int. J. Impact Eng., 35, 1441

Brown, P., Spalding, R. E., ReVelle, D. O., Tagliaferri, E., \& Worden, S. P. 2002, Nature, 420,294

Brown, P. G., Assink, J. D., Astiz, L., et al. 2013, Nature, 503, 238

Cavalié, T., Feuchtgruber, H., Lellouch, E., et al. 2013, A\&A, 553, A21

Christou, A. A. 2010, MNRAS, 402, 2759

Colina, L., Bohlin, R. C., \& Castelli, F. 1996, AJ, 112,307

Cook, A. F., \& Duxbury, T. C. 1981, J. Geophys. Res., 86, 8815

Crawford, D. A. 1997, Ann. N. Y. Acad. Sci., 822, 155

Delcroix, M., Hueso, R., Juaristi, J., \& Sanchez-Lavega, A. 2017, European Planetary Science Congress, 11, EPSC2017

de Pater, I., Fletcher, L. N., Pérez-Hoyos, S., et al. 2010, Icarus, 210, 722

Grasset, O., Dougherty, M. K., Coustenis, A., et al. 2013, Planet. Space Sci., 78, 1

Hammel, H. B., Beebe, R. F., Ingersoll, A. P., et al. 1995, Science, 267, 1288

Hammel, H. B., Wong, M. H., Clarke, J. T., et al. 2010, ApJ, 715, L150

Harrington, J., de Pater, I., Brecht, S. H., et al. 2004, Lessons from ShoemakerLevy 9 About Jupiter and Planetary Impacts, eds. F. Bagenal, T. E. Dowling, \& W. B. McKinnon, 159

Hueso, R., Legarreta, J., Pérez-Hoyos, S., et al. 2010a, Planet. Space Sci., 58, 1152

Hueso, R., Wesley, A., Go, C., et al. 2010b, ApJ, 721, L129

Hueso, R., Pérez-Hoyos, S., Sánchez-Lavega, A., et al. 2013, A\&A, 560, A55

Hueso, R., Juaristi, J., Legarreta, J., et al. 2018, Planet. Space Sci., 150, 22

Karkoschka, E. 1994, Icarus, 111, 174

Klekociuk, A. R., Brown, P. G., Pack, D. W., et al. 2005, Nature, 436, 1132

Korycansky, D. G., Harrington, J., Deming, D., \& Kulick, M. E. 2006, ApJ, 646, 642

Krüger, H., Grün, E., Hamilton, D. P., et al. 1998, Planet. Space Sci., 47, 85

Krüger, H., Krivov, A. V., \& Grün, E. 2000, Planet. Space Sci., 48, 1457

Krüger, H., Krivov, A. V., Sremčević, M., \& Grün, E. 2003, Icarus, 164, 170

Lellouch, E., Bézard, B., Moses, J. I., et al. 2002, Icarus, 159, 112

Levison, H. F., Duncan, M. J., Zahnle, K., Holman, M., \& Dones, L. 2000, Icarus, 143,415

Madiedo, J. M., Ortiz, J. L., Morales, N., \& Cabrera-Caño, J. 2015, Planet. Space Sci., 111, 105

Mendikoa, I., Sánchez-Lavega, A., Pérez-Hoyos, S., et al. 2016, PASP, 128, 035002

Mendikoa, I., Sánchez-Lavega, A., Pérez-Hoyos, S., et al. 2017, A\&A, 607, A72

Miller, S. D., Straka, W. C., Bachmeier, A. S., et al. 2013, Proc. Natl. Acad. Sci., 110,18092

Moses, J. I., \& Poppe, A. R. 2017, Icarus, 297, 33

Mousis, O., Hueso, R., Beaulieu, J.-P., et al. 2014, Exper. Astron., 38, 91

Palotai, C., Korycansky, D. G., Harrington, J., Rebeli, N., \& Gabriel, T. 2011, ApJ, 731, 3

Pérez-Hoyos, S., Sanz-Requena, J. F., Sánchez-Lavega, A., et al. 2012, Icarus, 221, 1061

Pond, J. W. T., Palotai, C., Gabriel, T., et al. 2012, ApJ, 745, 113

Poppe, A. R. 2016, Icarus, 264, 369

Porco, C. C., West, R. A., McEwen, A., et al. 2003, Science, 299, 1541

Salyk, C., Ingersoll, A. P., Lorre, J., Vasavada, A., \& Del Genio, A. D. 2006, Icarus, 185,430

Sánchez-Lavega, A., Gómez, J. M., Rojas, J. F., et al. 1998, Icarus, 131, 341

Sánchez-Lavega, A., Wesley, A., Orton, G., et al. 2010, ApJ, 715, L155

Sánchez-Lavega, A., Orton, G. S., Hueso, R., et al. 2011, Icarus, 214, 462

Sánchez-Lavega, A., Rojas, J. F., Hueso, R., et al. 2012, SPIE Conf. Ser., 8446

Schenk, P. M., Chapman, C. R., Zahnle, K., \& Moore, J. M. 2004, Ages and Interiors: The Cratering Record of the Galilean Satellites, eds. F. Bagenal, T. E. Dowling, \& W. B. McKinnon, 427

Selsis, F., Brillet, J., \& Rapaport, M. 2004, A\&A, 416, 783

Spencer, J. R., \& Mitton, J. 1995, The Great Comet Crash (CUP)

Sremčević, M., Krivov, A. V., Krüger, H., \& Spahn, F. 2005, Planet. Space Sci., 53,625

Tabe, I., Watanabe, J.-I., \& Jimbo, M. 1997, PASJ, 49, L1

Zahnle, K., Schenk, P., Levison, H., \& Dones, L. 2003, Icarus, 163, 263 